\title{
Dissociation of Fast Ions Analyzed by Time-of-Flight
}

\author{
C.R. Ponciano, R.C.C. Ladeia, V.M. Collado, and E.F. da Silveira* \\ Departamento de Física, Pontifícia Universidade Católica do Rio de Janeiro, \\ Rua Marquês de São Vicente, 225, CP 38071, Rio de Janeiro 22452-970, Brazil
}

Received on 9 May, 2001

\begin{abstract}
The fragmentation of metastable ions, having keV of kinetic energy, is analyzed by time-of-flight technique. Assuming isotropic distribution of fragments in a free field region, it is deduced an analytical expression to describe the corresponding peak shapes in linear TOF spectrometers. Metastable ion mean-life and the kinetic energy release (Q-value) are the quantities extracted from data fitting. As an illustration, the dissociation of $\mathrm{C}_{8} \mathrm{H}_{10} \mathrm{~N}^{+}$metastable ions, desorbed by ${ }^{252} \mathrm{Cf}$ fission fragment impact on organic target, is studied.
\end{abstract}

\section{Introduction}

Molecular dissociation has been investigated over decades by physicists and by chemists for reasons ranging from fundamental studies to practical applications. Such studies are important gateways for the understanding of molecular structure from an interatomic force description.

There are several possible agents to induce molecular dissociation, such as laser, synchrotron light, electron and ion beams, as well as many experimental techniques to analyze the produced fragments. These techniques are designed according to the specific characteristics of the projectile-target system: gaseous or solid target, absolute cross section of the phenomenon, selectivity of the excitation, molecular structure complexity, etc. Literature on this subject is immense, so that the following discussion will be restricted to metastable ion species dissociation; some basic references are 1-10.

In this article, it is considered the situation in which a fast single-charged metastable precursor dissociates into two stable fragments: one neutral and another single-charged ion. In contrast to ions produced usually in laboratory with a few eV of kinetic energy, fast ions are those moving in the keV-energy range. Under this aspect, metastable ions may be defined as unstable species that live long enough to become fast ions, surviving external high electric field acceleration and having their decay directly observed. By using a typical $\mathrm{kV} / \mathrm{mm}$ acceleration electric field, this means - in practice - that metastable ions have mean-lives larger than 100 ns. A method, based on time-of- flight (TOF) technique is developed to estimate $\tau$, the ion mean-life, and to determine $\mathrm{Q}$, the kinetic energy increase due to fragmentation. The analysis is then applied for the dissociation of desorbed ions from solid targets bombarded by $\mathrm{MeV}$ projectiles.

\section{PDMS and the experimental set-up}

The mass determination of the desorbed ions was done by a home-made linear PDMS spectrometer, Fig. 1. A very brief description of Plasma Desorption Mass Spectrometry [11] follows. A ${ }^{252} \mathrm{Cf}$ fission fragment source of about $20 \mu \mathrm{Ci}$ is used to induced metastable ion desorption from solid samples: while one fission fragment impacts the target, detection of the complementary fission fragment generates a start signal for the TOF acquisition system. The desorbed ions are accelerated in an uniform electric field region (length $d_{1}$ ) and launched towards a much longer electric field free region (length $L)$. If ion fragmentation does not occur in none of both regions, the ions are detected in the stop detector and their times of flight, $T_{m}$, are measured.

Defining $U$ as the extraction potential and $q$ as the ion charge, and considering that the initial kinetic energy $(\mathrm{eV})$ of the desorbed ions are negligible with respect to their final energy $\left(E_{f}=q U \approx 10 \mathrm{keV}\right)$, the mass of each ion species is given by

$$
m=\frac{2 E_{f}}{v^{2}}=2 q U\left(\frac{T_{m}}{2 d_{1}+L}\right)^{2}
$$

An ion filter close to the stop detector may be activated only when detection of neutral fragments are desired. In this case, the grid 3 potential $\left(U_{G 3}\right)$ must be greater than the sample potential $(U)$ and Eq. 1 holds only for neutrals. Detailed description of this PDMS spectrometer can be found in reference [12].

\footnotetext{
*author for correspondence: enio@vdg.fis.puc-rio.br
} 


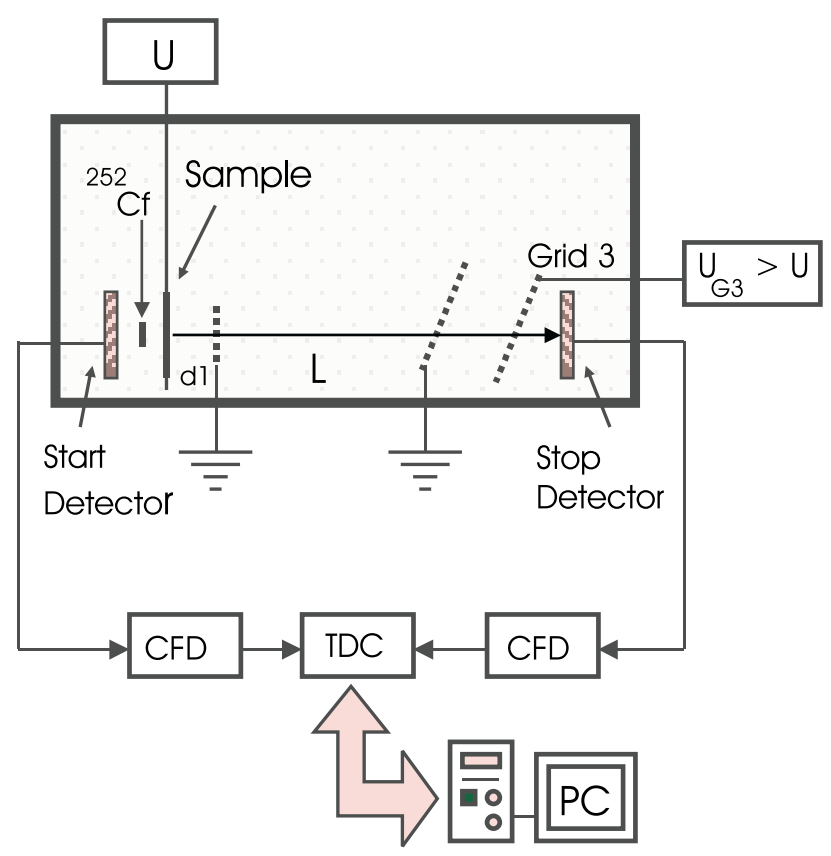

Figure 1. Diagram of a ${ }^{252} \mathrm{Cf}$ - PDMS time-of-flight mass spectrometer. $U$ is the target potential and $U_{G 3}$ is the ion filter repelling potential. $d_{1}=0.70 \mathrm{~cm}$ and $L=134 \mathrm{~cm}$ are the acceleration and free field region lengths. The time interval between the start and the stop signals is measured by the TDC unit. The electronic devices are: CFD - Constant Fraction Discriminator; TDC - Time Digital Converter; PC - Personal Computer.

\section{The fragmentation analysis}

According to the previous definition, the metastable ion species mean life, $\tau$, is comparable or longer than the traversing time over the acceleration region

$$
t_{1}=d_{1} \sqrt{\frac{2 m}{q U}}
$$

Moreover, if $\tau$ it is shorter than its total time of flight $T_{m}$, a substantial fraction of the desorbed metastable ions disintegrates in the field free region. The binary process $m^{+} \rightarrow m_{0}+m_{1}^{+}$is the most likely to occur and the kinetic energy excess, $Q$, appears after fragmentation:

$$
Q=\left(\frac{1}{2} m_{0} v_{0}^{2}+\frac{1}{2} m_{1} v_{1}^{2}\right)-\frac{1}{2} m v^{2}
$$

where, in the laboratory frame, $v_{0}$ and $v_{1}$ are the velocities of the neutral and charged fragments and $v$ is the precursor metastable ion velocity in the free field region.

In the precursor ion frame, the direction of the fragmentation axis is distributed isotropically and the fragment velocities $\Delta v$ are promptly determined by linear momentum conservation:

$$
\Delta v_{0}=\sqrt{\frac{2 m_{1}}{m m_{0}} Q}
$$

$$
\Delta v_{1}=\sqrt{\frac{2 m_{0}}{m m_{1}} Q}
$$

If fragmentation occurs at the instant $t_{f}$ after desorption, the distance precursor ion to the detector is $L-v\left(t_{f}-t_{1}\right)$. Therefore, the arrival times of the neutral and charged fragments at the detector are respectively

$$
\begin{gathered}
T_{0}=t_{f}+\frac{L-v\left(t_{f}-t_{1}\right)}{v+\Delta v_{0} \cos \theta} \\
T_{1}=t_{f}+\frac{L-v\left(t_{f}-t_{1}\right)}{v+\Delta v_{1} \cos (\pi+\theta)}
\end{gathered}
$$

where $\theta$ is the angle between the neutral fragment velocity, in the precursor ion frame, and the spectrometer axis.

Integration over $\theta$ generates the time-of-flight distribution $n(T)=d N / d T$ for the fragments, which shape depends directly on $Q$ : larger is $Q$, broader is the TOF peak. This integral should be performed very carefully for two reasons: i) the instant $t_{f}$ is not fixed, but belongs to an exponential decay-type probabilistic distribution; ii) in the precursor frame, the detector is moving towards the fragments. This fact brings mathematical difficulties, which are partially solved if one considers that $\Delta v \ll v$.

For an ensemble of fragmentation events occurring between $t_{f}$ and $t_{f}+d t_{f}$, the neutral and ion fragments will be dispersed isotropically in two expanding spherical shells centered in the precursor center of mass. For each shell, the number of fragments emitted in the solid angle $d \Omega$ is

$$
d\left(\frac{d N}{d t_{f}}\right)=\frac{d \Omega}{4 \pi} n_{f}\left(t_{f}\right)
$$

where

$$
n_{f}\left(t_{f}\right)=\frac{N_{0}}{\tau} e^{-t_{f} / \tau}
$$

is the fragmentation rate at instant $t_{f}$. Our goal is to calculate the number of particles, for each fragment species, $d N$, arriving between $T$ and $T+d T$. The distribution $d N / d T$ is the quantity to be compared to the experimental peak shape, which is directly provided by the acquisition system. During the time interval $d T$, the fragments of each shell, produced between $t_{f}$ and $t_{f}+d t_{f}$, reach the detector inside a ring corresponding to $d \Omega=2 \pi \operatorname{sen} \theta d \theta$ and in a rate

$$
\frac{d^{2} N}{d T d t_{f}}=\frac{1}{4 \pi} n_{f}\left(t_{f}\right) 2 \pi \operatorname{sen} \theta d \theta
$$

This expression needs to be integrated over $t_{f}$, within limits compatible with fragment detection at instant $T$, and a $t_{f}^{\max }$ must be determined for each arrival time. For example, it is not possible that the fragment arrival times be very different from $T_{m}$ if fragmentation occurs close to the detector. Imposing $\theta=0$ in Eq. 5, one gets:

$$
t_{f}^{\max }=T-\frac{v}{\Delta v}\left|T-T_{m}\right|
$$


In this expression, one should use $\Delta v=\Delta v_{0}$ or $\Delta v=$ $\Delta v_{1}$ respectively for the neutral or ion species. The integration of Eq. 8 becomes, after some mathematical manipulation using Eqs. 5 and 7:

$$
\frac{d N}{d T}=\frac{v}{2 \Delta v} \frac{N_{0}}{\tau} \int_{t_{1}}^{t_{f}^{\max }} e^{-t_{f} / \tau} \frac{T_{m}-t_{f}}{\left(T-t_{f}\right)^{2}} d t_{f}
$$

for each fragment species.

If $\tau \gg t_{f}^{\max }$, the expression 10 can be integrated analytically, given a peak shape which has a logarithmtype divergence at $T=T_{m}$. If $\tau \sim t_{1}$, the peak shape is close to a $1 /\left(T-t_{1}\right)^{2}$ distribution, keeping the singularity at $T=T_{m}$.

It can be seen from these expressions that the neutral and ion fragment peaks appear at the same position as the non-fragmented precursor peak, so that experimentally one observes the overlapping of the three peaks. To simplify the experimental analysis, one may introduce an ion filter just before the detector and let only neutral fragments to be detected. It is also possible to deflected the flying ions into a second detector and separate the precursor ion peak from the ion fragment one. Eqs. 4, 9 and 10 also show that the fragment peak shapes depend on the ion fragment and precursor mass ratio. Therefore, if $m_{1}$ is not known, it may also used as free parameter in the peak shape analysis.

\section{Results and discussion}

In order to understand the effects of $\tau, Q$ and $m_{1} / m$ on the Eq. 10 theoretical predictions, simulations corresponding to possible real configurations are presented in Fig. 2 for $m=120 u, q U=15 \mathrm{keV}, d_{1}=0.70 \mathrm{~cm}$ and $L=134 \mathrm{~cm}$. The obtained neutral fragment peak shape are respectively shown in Figs. 2a, 2b and 2c. General comments are:

i) The curves are fairly symmetric around $T=T_{m}$. Fragmentation events with $T<T_{m}$ correspond to neutral emission towards the detector (neutral fragments are faster than their precursor), while $T>T_{m}$ ones refer to ion fragment emission in this direction.

ii) There are two basic contributions: one, produced by dissociation far from the detector and generating a very broad distribution; the other one is produced by dissociations close to the detector and generating a pronounced peak.

iii) Longer $\tau$ is, less dissociations occur in the field free region and less clear is the border between the broad and the peak contributions (Fig. 2a).

iv) Larger is the $Q$-value or the ion fragment mass, broader the $d N / d T$ distribution is, and lower is the background level (Figs. 2b and $2 \mathrm{c}$ ). In fact, note that in Eq. $4 \mathrm{a}, \Delta v_{0}$ increases with the product $m_{1} Q$.
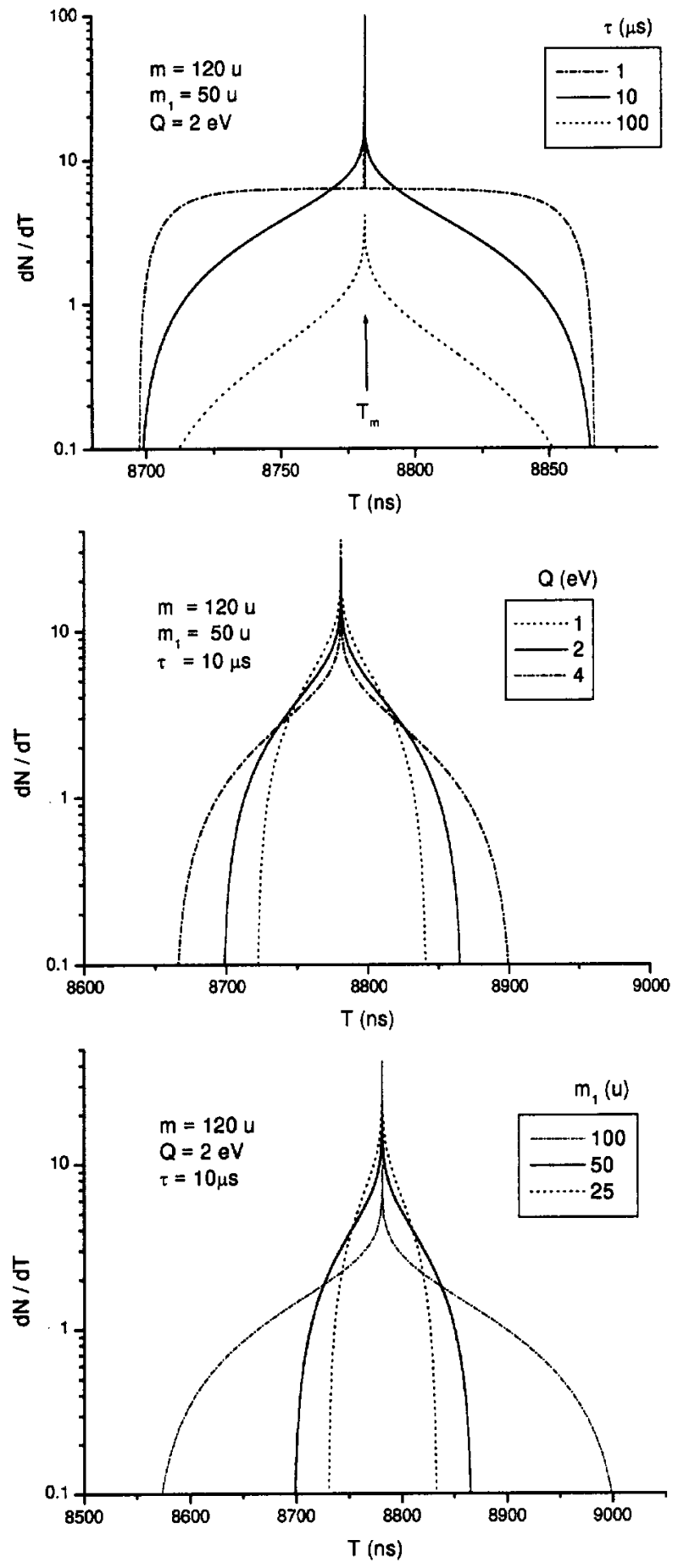

Figure 2. Simulations based on Eq. 10, showing respectively in a, b and c, the $\tau, Q$ and $m_{1}$ parameter effects on the TOF peak shape of neutral fragments. The considered precursor ion dissociation is $120^{+} \rightarrow m_{1}^{+}+m_{0}$. The extraction potential is $15 \mathrm{kV}$ and the spectrometer lengths are $d_{1}$ $=0.70 \mathrm{~cm}$ and $L=134 \mathrm{~cm}$. A singularity occurs at $T_{m}=$ $8781 \mathrm{~ns}$, the non-fragmented precursor TOF.

Predictions are now compared with experimental data obtained from the fission fragment bombardment on phenylalanine target (molecular mass $M=165 \mathrm{u}$ ). An ion filter was employed, so that only the neutral fragments of desorbed positive ion precursors were al- 
lowed to be detected in the linear spectrometer. The obtained neutral fragment TOF spectrum is presented in Fig. 3, in which the most prominent peak refers to mass $120 \mathrm{u}$ precursor $\left(\mathrm{C}_{8} \mathrm{H}_{10} \mathrm{~N}^{+}\right)$. It has been suggest [12], from TOF-coincidence measurements, that the tropylium ion $\left(m_{1}=91 \mathrm{u}, \mathrm{C}_{7} \mathrm{H}_{7}^{+}\right)$formation is the pathway for the $120^{+}$fragmentation. Assuming such fragmentation, $Q=1.0 \mathrm{eV}$ and $\tau \sim 50 \mu$ s were the best parameter values in the data fitting with Eq. 10, as illustrated in Fig. 4. It should be mentioned that no modification in the $t_{f}^{\max }$ expression (Eq. 9) was introduced due to the activated ion filter, which suppresses fragmentation in the region between it and the detector.

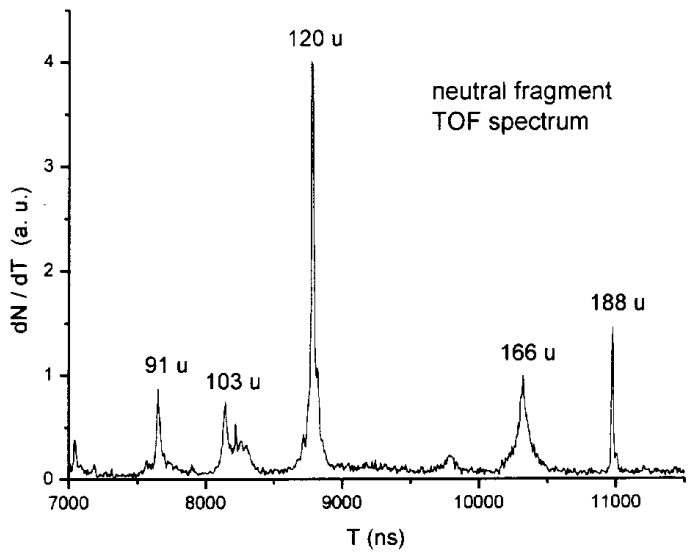

Figure 3. Neutral fragment TOF spectrum of the desorbed positive ions from a phenylalanine sample. $U=15 \mathrm{kV}$ and $U_{G 3}=17.6 \mathrm{kV}$. The $120 \mathrm{u}$ is the mass of the single-charge precursor ion which dissociation is being investigated. The spectrometer mass resolution can be appreciated from the $m=188$ u mass peak width.

\section{Conclusions}

The dynamics of binary fragmentation of a free metastable ion in TOF linear spectrometer is described by analytical expressions and peak shapes of neutral and ion fragment peaks are predicted. The method was applied to the neutral fragment peak shape fitting of the $\mathrm{C}_{8} \mathrm{H}_{10} \mathrm{~N}^{+}$metastable ion decay, assuming the $120^{+} \rightarrow 91^{+}+29^{\circ}$ pathway. A good agreement with our experimental data was found for $Q=1.5 \mathrm{eV}$ and $\tau \sim 50 \mu$ s parameters, giving confidence that the model is able to describe the phenomenon.

\section{Acknowledgements}

$\mathrm{CNPq}$, Faperj and PADCT III are gratefully acknowledged by their partial support to this work. R.C.C. Ladeia thanks PIBIC for her scholarship.

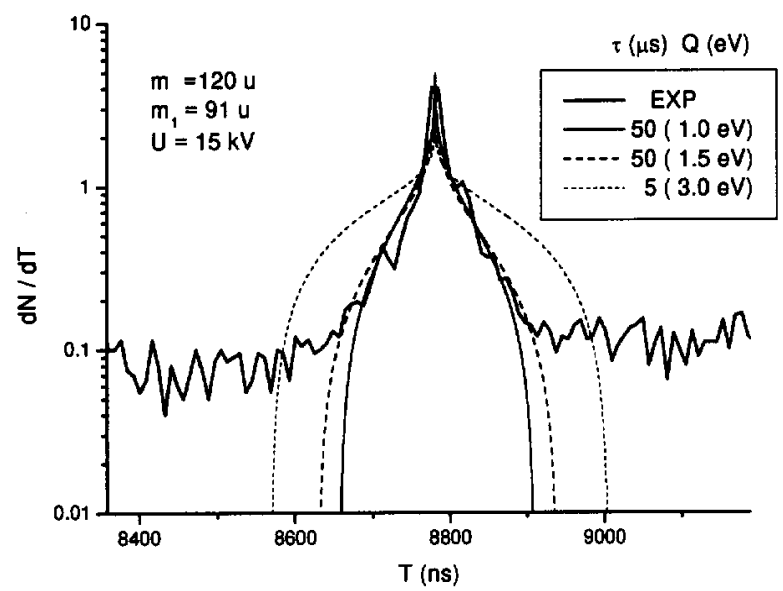

Figure 4. Comparison of the 120 peak shape, selected from the neutral fragment TOF spectrum of Fig. 3, with Eq. 10 theoretical predictions. Results from three set of parameter values are shown.

\section{References}

[1] R.G. Cooks, J.H. Beynon, R. M. Caprioli and G. R. Lester, Metastable Ions, Elsevier Scientific Publishing Company, 1973.

[2] W.F. Haddon and F. W. McLafferty, Analytical Chem. 41, 31 (1969).

[3] F.W. McLafferty, Interpretation of Mass Spectra, University Science Books, 3rd Edition, 1980.

[4] B.T. Chait, Int. J. Mass Spectrom. Ion Phys. 53, 227 (1983).

[5] S. Della-Negra and Y. Le Beyec, Anal. Chem. 57, 2035 (1985).

[6] A. Schueler, R. Beavis, G. Bolbach, W. Ens, D.E. Main and K.G. Standing, Springer Ser.Chem. Phys. 44, 57 (1986).

[7] X. Tang, R. Beavis, W. Ens, F. Lafortune, B. Schueler and K.G. Standing, Int. J. Mass Spectrom. Ion Processes, 85, 43 (1988).

[8] H.J. Neusser, Int. J. Mass Spectrom. Ion Processes, 79, 141 (1987).

[9] P.A. Demirev, Mass Spectrometry Reviews, 14, 279 (1995).

[10] R.J. Cotter, Time-of-Flight Mass Spectrometry - Instrumentation and Applications in Biological Research, ACS Professional Reference Books, 1997.

[11] R.D. Macfarlane and D.F. Torgerson, Int. J. Mass Spectrom. Ion Phys. 21, 81 (1976).

[12] C.R. Ponciano, Doctoral Dissertation, Pontifícia Universidade Católica, Rio de Janeiro, 1996. 